Ann. Inst. Statist. Math.

Vol. 47, No. 1, 129-136 (1995)

\title{
RESIDUALS IN THE GROWTH CURVE MODEL
}

\author{
DIETRICH VON ROSEN \\ Department of Mathematics, Uppsala University, Box 480, S-751 06 Uppsala, Sweden
}

(Received April 8, 1994; revised October 11, 1994)

\begin{abstract}
Residuals for the Growth Curve model will be discussed. In univariate linear models as well as the ordinary multivariate analysis of variance model residuals are based on the difference between the observations and the mean whereas for the Growth Curve model we have three different residuals all showing various aspects useful for validating analysis. For these residuals some basic properties are established.
\end{abstract}

Key words and phrases: GMANOVA, Growth Curve model, residuals.

\section{Introduction}

In this paper we will discuss residuals in the Growth Curve model due to Potthoff and Roy (1964). For more details we refer to von Rosen (1994) where the results, via Edgeworth type expansions, are applied to a real data set. According to the terminology put forward in von Rosen (1989) we will refer to the model as a $M L N M(A B C)$. The reason for this notation follows from the next definition as well as from some extensions presented in von Rosen (1989).

Definition 1.1. The $M L N M(A B C)$. Let $X: p \times n, A: p \times q q \leq p, B: q \times k$, $C: k \times n \rho(C)+p \leq n$ and $\Sigma: p \times p$ is positive definite. The columns of $X$ are independently $p$-variate normally distributed with an unknown dispersion matrix $\Sigma$ and $E[X]=A B C$, where $A$ and $C$ are known design matrices and $B$ is an unknown parameter matrix.

In the definition as well as in the sequel $\rho(\cdot)$ denotes the rank. The $M L N M(A B C)$ is an extension of the ordinary multivariate analysis of variance model and is applicable when a linear mean structure exists within the experimental units. There exist many fields where the $M L N M(A B C)$ has been applied. In particular the model is useful for analysing short time series of repeated measurements when little knowledge about the covariance structure is available. For example, growth curves. Fundamental to all analysis with the $M L N M(A B C)$ are the interpretation of the design matrices $A$ and $C$. In our setting $A$ models the within individuals structure, i.e. the repeated measurements on each experimental unit, and $C$ models the between individuals structure, i.e. $C$ is the same design 
matrix as in univariate linear models and ordinary multivariate analysis of variance. For reviews of the model and related works see Woolson and Leeper (1980), Seber (1984) or von Rosen (1991). Maximum likelihood estimators for the parameters in the $M L N M(A B C)$ are given by (e.g. see Srivastava and Khatri (1979) or von Rosen (1989))

$$
\hat{B}=\left(A^{\prime} S^{-1} A\right)^{-} A^{\prime} S^{-1} X C^{\prime}\left(C C^{\prime}\right)^{-}+\left(A^{\prime}\right)^{o} Z_{1}+A^{\prime} Z_{2} C^{o^{\prime}},
$$

where $Z_{1}$ and $Z_{2}$ are arbitrary matrices,

$$
S=X\left(I-C^{\prime}\left(C C^{\prime}\right)^{-} C\right) X^{\prime}
$$

and

$$
\begin{aligned}
n \hat{\Sigma} & =(X-A \hat{B} C)(X-A \hat{B} C)^{\prime} \\
& =S+S A^{o}\left(A^{o^{\prime}} S A^{o}\right)^{-1} A^{o^{\prime}} X C^{\prime}\left(C C^{\prime}\right)^{-} C X^{\prime} A^{o}\left(A^{o^{\prime}} S A^{o}\right)^{-1} A^{o^{\prime}} S .
\end{aligned}
$$

In (1.1) and (1.3) we have used the notation $A^{\circ}$ for any matrix of full rank which is spanning the orthogonal complement to $\mathcal{C}(A)$, i.e. $\mathcal{C}\left(A^{o}\right)=\mathcal{C}(A)^{\perp}$ where $\mathcal{C}(A)$ stands for the linear space generated by the columns of $A$ (column vector space, range space). The matrix $A^{\circ}$ is not unique but all results presented in this paper will be invariant with respect to the choice of $A^{\circ}$ (see formula 2.4 given below). Furthermore, in (1.1)-(1.3), - stands for an arbitrary $g$-inverse in the sense of $G G^{-} G=G$. The estimator $\hat{\Sigma}$ is always unique and $\hat{B}$ is unique if $A$ and $C$ are of full rank. Since (1.1) is a weighted estimator with a random weight $S^{-1}(1.1)$ is more difficult to handle than univariate least squares estimators. Furthermore, note that

$$
A \hat{B} C=A\left(A^{\prime} S^{-1} A\right)^{-} A^{\prime} S^{-1} X C^{\prime}\left(C C^{\prime}\right)^{-} C
$$

which is always unique.

\section{Residuals}

In principle, when doing inference there are mainly two different strategies. One is to require as few assumptions as possible for the data, leading to so called robust methods. The other approach is to find models and then inference is based on these models together with diagnostic tools for validating the model. When considering univariate linear models many diagnostic tools are based on residuals. There exist many types of residuals, e.g. ordinary residuals, studentized residuals, external residuals, internal residuals (see Belsley et al. (1980), Cook and Weisberg (1982)), recursive residuals (Tobing and McGilchrist (1992)), as well as others. However, for multivariate linear models very few results exist and for the $M L N M(A B C)$, to our knowledge, any discussion of residuals does not exist. Hence, if using the $M L N M(A B C)$ in practise, it is important to fill this gap and indeed, some completely new problems arise. 
In the univariate linear model, $X=\beta C+\epsilon$, the residuals are obtained if projecting $X$ on $\mathcal{C}\left(C^{\prime}\right)^{\perp}$, i.e. $X\left(I-C^{\prime}\left(C C^{\prime}\right)^{-} C\right)$. In the $M L N M(A B C)$ there are two spaces of interest, namely $\mathcal{C}_{\Sigma}(A)$ and $\mathcal{C}(C)$, or more precisely the tensor product of these, i.e. $\mathcal{C}\left(C^{\prime}\right) \otimes \mathcal{C}_{\Sigma}(A)$. Here $\mathcal{C}_{\Sigma}(A)$ means that we have an inner product which is defined by aid of $\Sigma^{-1}$, i.e. $(x, y)=x^{\prime} \Sigma^{-1} y$ and $\mathcal{C}(A)=\mathcal{C}_{I}(A)$. Unfortunately, for the $M L N M(A B C), \Sigma$ is unknown, but we see that the maximum likelihood estimators, given by (1.1) and (1.3), respectively, are build up with the help of projectors where the inner product is based on $S^{-1}$. Hence, maximum likelihood theory tells us that we can replace $\Sigma$ by $S$. Furthermore, from (1.4) follows that $A \hat{B} C$ is obtained with the help of the projection of $X$ on $\mathcal{C}\left(C^{\prime}\right) \otimes \mathcal{C}_{S}(A)$. In order to study residuals according to ideas for univariate linear models we will study $\left(\mathcal{C}\left(C^{\prime}\right) \otimes \mathcal{C}_{S}(A)\right)^{\perp}$, i.e. residuals are defined on the space which is orthogonal to the space generated by the design matrices. Typically for the $M L N M(A B C)$ is that $\left(\mathcal{C}\left(C^{\prime}\right) \otimes \mathcal{C}_{S}(A)\right)^{\perp}$ consists of three orthogonal spaces:

$$
\left(\mathcal{C}\left(C^{\prime}\right) \otimes \mathcal{C}_{S}(A)\right)^{\perp}=\mathcal{C}\left(C^{\prime}\right)^{\perp} \otimes \mathcal{C}_{S}(A)^{\perp} \boxplus \mathcal{C}\left(C^{\prime}\right)^{\perp} \otimes \mathcal{C}_{S}(A) \boxplus \mathcal{C}\left(C^{\prime}\right) \otimes \mathcal{C}_{S}(A)^{\perp},
$$

where $\boxplus$ stands for the orthogonal sum. Hence the following residuals are obtained;

$$
\begin{aligned}
& R_{1}=S A^{o}\left(A^{o^{\prime}} S A^{o}\right)^{-} A^{o^{\prime}} X\left(I-C^{\prime}\left(C C^{\prime}\right)^{-} C\right), \\
& R_{2}=A\left(A^{\prime} S^{-1} A\right)^{-} A^{\prime} S^{-1} X\left(I-C^{\prime}\left(C C^{\prime}\right)^{-} C\right), \\
& R_{3}=S A^{o}\left(A^{o^{\prime}} S A^{o}\right)^{-} A^{o^{\prime}} X C^{\prime}\left(C C^{\prime}\right)^{-} C,
\end{aligned}
$$

where $R_{1}$ is obtained from the space $\mathcal{C}\left(C^{\prime}\right)^{\perp} \otimes \mathcal{C}_{S}(A)^{\perp}, R_{2}$ from $\mathcal{C}\left(C^{\prime}\right)^{\perp} \otimes \mathcal{C}_{S}(A)$ and $R_{3}$ from $\mathcal{C}\left(C^{\prime}\right) \otimes \mathcal{C}_{S}(A)^{\perp}$. If we for simplicity just will look at $R_{1}+R_{2}$ and $R_{3}$ the interpretation is fairly clear. $R_{1}+R_{2}=X\left(I-C^{\prime}\left(C C^{\prime}\right)^{-} C\right)$ represents the difference between the observation and $X C^{\prime}\left(C C^{\prime}\right)^{-} C$ (the mean) and $R_{3}$ reflects the difference between the mean and the estimated model $A \hat{B} C$, since (2.3) is identical to $R_{3}=X C^{\prime}\left(C C^{\prime}\right)^{-} C-A \hat{B} C$ which follows from the fact that

$$
I-A\left(A^{\prime} S^{-1} A\right)^{-} A^{\prime} S^{-1}=S A^{o}\left(A^{o^{\prime}} S A^{o}\right)^{-} A^{o^{\prime}} .
$$

However, we recommend that $R_{1}$ and $R_{2}$ should be calculated separately because elements in these matrices may appear with opposite sign and of the same size and then elements in $R_{1}+R_{2}$ will be close to zero.

Remember that $\mathcal{C}_{\Sigma}(A)$ represents the within individuals structure. Hence, if studing $R_{1}$ and $R_{2}$ separately we note that $R_{2}$ stands for the projection of the difference between the observation and the mean on $\mathcal{C}_{S}(A)$ whereas $R_{1}$ stands for the projection of the difference between the observation and the mean on $\mathcal{C}_{S}(A)^{\perp}$. This means that both $R_{1}$ and $R_{3}$ mirror the within individuals model assumption whereas $R_{1}$ and $R_{2}$ can be used to investigate the between individuals assumptions. 


\section{Basic properties}

Unfortunately the distribution for $\left(R_{1}, R_{2}, R_{3}\right)$ is difficult to obtain as well as the marginal distributions for $R_{i}, i=1,2,3$. Therefore we will be concentrating on moment relations. These give us a possibility to understand the estimators as well as to approximate the distribution in a convenient manner. Indeed, in practise, already for the univariate linear model the complete distribution for the residuals is not always utilized. For instance, residuals are often treated as if they are independently distributed which is not the case. The most elementary properties for $R_{1}, R_{2}, R_{3}$ are presented in our first theorem and especially for graphical representations of residuals they may be important. Let $C_{r}\left[R_{i}\right]$ represent the cumulants of $R_{i}$ of $r$-th order and in particular $C_{1}\left[R_{i}\right]=E\left[R_{i}\right]$ and $C_{2}\left[R_{i}\right]=$ $D\left[R_{i}\right]=E\left[\operatorname{vec}\left(R_{i}-E\left[R_{i}\right]\right) \operatorname{vec}^{\prime}\left(R_{i}-E\left[R_{i}\right]\right)\right]$. In the theorem $\stackrel{k}{\otimes} R_{i}$ stands for

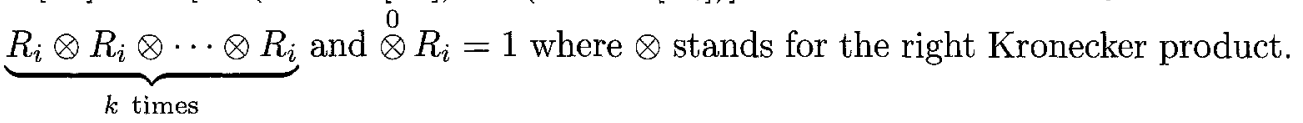

THEOREM 3.1. Let $R_{1}, R_{2}$ and $R_{3}$, respectively be given by (2.1), (2.2) and (2.3). Then

(i) $E\left[R_{i}\right]=0, i=1,2,3$,

(ii) $E\left[\stackrel{r}{\otimes} R_{i}\right]=0, i=1,2,3$ for odd $r$,

(iii) $C_{r}\left[R_{i}\right]=0, i=1,2,3$ for odd $r$.

Proof. $\quad R_{i}, i=1,2$, are odd functions in $X\left(I-C^{\prime}\left(C C^{\prime}\right)^{-} C\right)$ which in turn is normally distributed with mean zero. Since the normal distribution is symmetric $E\left[R_{i}\right]=0, i=1,2$. For $R_{3}$ it is noted that $X C^{\prime}\left(C C^{\prime}\right)^{-} C$ is independent of $S$ and since $E\left[A^{o^{\prime}} X C^{\prime}\left(C C^{\prime}\right)^{-} C\right]=0$ we obtain that $E\left[R_{3}\right]=0$. These results can immediately be generalized to cover moments of odd order, i.e. $E\left[\stackrel{r}{\otimes} R_{i}\right]=0, i=$ $1,2,3$, for odd $r$. Furthermore, by the correspondence of moments and cumulants (iii) follows from (ii).

One consequence of the theorem is that the distribution for the residuals are symmetric.

In the subsequent it will be convenient to rewrite $R_{i}$ in a canonical form and we will use the following representation;

$$
A^{o^{\prime}}=T\left(I_{p-\rho(A)}: 0\right) \Gamma \Sigma^{-1 / 2}
$$

where $T$ is non-singular, $\Gamma^{\prime}=\left(\Gamma_{1}^{\prime}: \Gamma_{2}^{\prime}\right) p \times p-\rho(A): p \times \rho(A)$ is orthogonal and $\Sigma^{1 / 2}$ is supposed to be symmetric. Furthermore let, $Y \sim N_{p, n}\left(0, I, I-C^{\prime}\left(C C^{\prime}\right)^{-} C\right)$, $Y^{\prime}=\left(Y_{1}^{\prime}: Y_{2}^{\prime}\right) n-\rho(C) \times p-\rho(A): n-\rho(C) \times \rho(A)$ and $Z \sim N_{p, n}\left(0, I, C^{\prime}\left(C C^{\prime}\right)^{-} C\right)$. Observe that $Z$ and $Y$ are independent and that also $Y_{1}$ and $Y_{2}$ are independent. Using these definitions we obtain from (2.1), (2.2) and (2.3) canonical representations of the residuals, i.e.

$$
\begin{aligned}
& R_{1}^{\prime} \sim Y_{1}^{\prime}\left(Y_{1} Y_{1}^{\prime}\right)^{-1} Y_{1} Y^{\prime} \Gamma \Sigma^{1 / 2}=\left\{Y_{1}^{\prime}: Y_{1}^{\prime}\left(Y_{1} Y_{1}^{\prime}\right)^{-1} Y_{1} Y_{2}^{\prime}\right\} \Gamma \Sigma^{1 / 2} \\
& R_{2}^{\prime} \sim\left(I-Y_{1}^{\prime}\left(Y_{1} Y_{1}^{\prime}\right)^{-1} Y_{1}\right) Y^{\prime} \Gamma \Sigma^{1 / 2}=\left(I-Y_{1}^{\prime}\left(Y_{1} Y_{1}^{\prime}\right)^{-1} Y_{1}\right) Y_{2}^{\prime} \Gamma_{2} \Sigma^{1 / 2} \\
& R_{3}^{\prime} \sim Z^{\prime}\left(Y_{1} Y_{1}^{\prime}\right)^{-1} Y_{1} Y^{\prime} \Gamma \Sigma^{1 / 2}=Z^{\prime}\left\{I:\left(Y_{1} Y_{1}^{\prime}\right)^{-1} Y_{1} Y_{2}^{\prime}\right\} \Gamma \Sigma^{1 / 2}
\end{aligned}
$$


where $\sim$ stands for equality in distribution. Furthermore, for later use note that

$$
\begin{aligned}
& \Sigma^{1 / 2} \Gamma_{1}^{\prime} \Gamma_{1} \Sigma^{1 / 2}=\Sigma-A\left(A^{\prime} \Sigma^{-1} A\right)^{-} A^{\prime} \quad \text { and } \\
& \Sigma^{1 / 2} \Gamma_{2}^{\prime} \Gamma_{2} \Sigma^{1 / 2}=A\left(A^{\prime} \Sigma^{-1} A\right)^{-} A^{\prime} .
\end{aligned}
$$

The next theorem includes the covariance matrix between two matrices $U$ and $V$ which is given by $C[U, V]=E\left[\operatorname{vec}(U) \operatorname{vec}(V)^{\prime}\right]-E[\operatorname{vec}(U)] E\left[\operatorname{vec}(V)^{\prime}\right]$ where the vec-operator is the operator defined by vec: $R^{n \times m} \rightarrow R^{n m}, x y^{\prime} \rightarrow y \otimes x$ for vectors $x$ and $y$ of size $n$ and $m$, respectively.

ThEOREM 3.2. Let $R_{1}, R_{2}$ and $R_{3}$, respectively be given by (2.1), (2.2) and (2.3). Then $C\left[R_{i}, R_{j}\right]=0, i \neq j, i, j=1,2,3$.

Proof. From (3.1) and (3.2) follows since $Y_{1}$ and $Y_{2}$ are independent that

$$
\begin{aligned}
C\left[R_{1}, R_{2}\right]= & E\left[\operatorname{vec}\left(\Sigma^{1 / 2} \Gamma_{1}^{\prime} Y_{1}\right) \operatorname{vec}^{\prime}\left\{\Sigma^{1 / 2} \Gamma_{2}^{\prime} Y_{2}\left(I-Y_{1}^{\prime}\left(Y_{1} Y_{1}^{\prime}\right)^{-1} Y_{1}\right)\right\}\right] \\
& +E\left[\operatorname{vec}\left(\Sigma^{1 / 2} \Gamma_{2}^{\prime} Y_{2} Y_{1}^{\prime}\left(Y_{1} Y_{1}^{\prime}\right)^{-1} Y_{1}\right) \operatorname{vec}^{\prime}\left(\Sigma^{1 / 2} \Gamma_{2}^{\prime} Y_{2}\left(I-Y_{1}^{\prime}\left(Y_{1} Y_{1}^{\prime}\right)^{-1} Y_{1}\right)\right)\right] \\
= & 0+E\left[\left(Y_{1}^{\prime}\left(Y_{1} Y_{1}^{\prime}\right)^{-1} Y_{1}\right)\left(I-Y_{1}^{\prime}\left(Y_{1} Y_{1}^{\prime}\right)^{-1} Y_{1}\right)\right] \otimes \Sigma^{1 / 2} \Gamma_{2} \Gamma_{2}^{\prime} \Sigma^{1 / 2}=0 .
\end{aligned}
$$

We also have, because $S$ and $X C^{\prime}\left(C C^{\prime}\right)^{-} C$ are independent and $E[X]=A B C$, that

$$
\begin{aligned}
C\left[R_{2}, R_{3}\right]= & E\left[\operatorname{vec}\left\{A\left(A^{\prime} S^{-1} A\right)^{-} A^{\prime} S^{-1} X\left(I-C^{\prime}\left(C C^{\prime}\right)^{-} C\right)\right\}\right. \\
& \left.\times E\left[\operatorname{vec}^{\prime}\left\{X C^{\prime}\left(C C^{\prime}\right)^{-} C\right\}\right]\left(I \otimes A^{o}\left(A^{o^{\prime}} S A^{o}\right)^{-1} A^{o^{\prime}} S\right)\right]=0 .
\end{aligned}
$$

The proof that $C\left[R_{1}, R_{3}\right]=0$ is identical.

In Theorem 3.2 we have established that $R_{1}, R_{2}$ and $R_{3}$ are uncorrelated. It would be of advantage for the interpretation of the values of the residuals if the residuals also are independently distributed. However, the next lines show that this is not the case. If $R_{1}$ and $R_{2}$ are independent they must be normally distributed because $R_{1}+R_{2}=X\left(I-C^{\prime}\left(C C^{\prime}\right)^{-} C\right)$ is normally distributed which is a well known characteristic of the class of normal distributions. We are going to show that $R_{2}$ is not normally distributed and from (3.2) follows that it is enough to show that $Y_{2} Y_{1}^{\prime}\left(Y_{1} Y_{1}^{\prime}\right)^{-} Y_{1}$ is not normally distributed which also confirms that $R_{1}$ can not be normally distributed. It would indeed be very suprising if this expression is normally distributed. Put

$$
H=E\left[\stackrel{2}{\otimes} Y_{2} Y_{1}^{\prime}\left(Y_{1} Y_{1}^{\prime}\right)^{-} Y_{1}\right]
$$

and if $Y_{2} Y_{1}^{\prime}\left(Y_{1} Y_{1}^{\prime}\right)^{-} Y_{1}$ is normally distributed the fourth ordered moments are related to $H$ in the following manner;

$$
\begin{aligned}
& E\left[\stackrel{4}{\otimes} Y_{2} Y_{1}^{\prime}\left(Y_{1} Y_{1}^{\prime}\right)^{-} Y_{1}\right] \\
& \quad=\stackrel{2}{\otimes H} H+\left(I_{r} \otimes K_{r, r} \otimes I_{r}\right)(\stackrel{2}{\otimes} H)\left(I_{s} \otimes K_{s, s} \otimes I_{s}\right)+K_{r, r^{3}}\left(2^{2} H\right) K_{s^{3}, s}
\end{aligned}
$$


where $K_{u, v}$ stands for the commutation matrix (see Magnus and Neudecker (1979)), $r=p-\rho(A)$ and $s=n-\rho(C)$. However, since $Y_{2}$ is normally distributed and is independent of $Y_{1}$ this is only true if

$$
E\left[\stackrel{2}{\otimes} Y_{1}^{\prime}\left(Y_{1} Y_{1}^{\prime}\right)^{-} Y_{1}\right]=\stackrel{2}{\otimes} E\left[Y_{1}^{\prime}\left(Y_{1} Y_{1}^{\prime}\right)^{-} Y_{1}\right]
$$

which is not the case. To show that $R_{1}$ and $R_{3}$ are not independent we may note that

$$
E\left[R_{1} R_{1}^{\prime} \otimes R_{3} R_{3}^{\prime}\right] \neq E\left[R_{1} R_{1}^{\prime}\right] \otimes E\left[R_{3} R_{3}^{\prime}\right] .
$$

Similar calculations also give that $R_{2}$ and $R_{3}$ can not be independent. However, note that $R_{1} R_{1}^{\prime}$ and $R_{2} R_{2}^{\prime}$ are independent. As already mentioned we will in this paper not obtain the exact distributions of the residuals because they are complicated. The only simple distribution property which we are able to obtain is that $R_{2} R_{2}^{\prime}$ is Wishart distributed, i.e. $W_{p}\left(A\left(A^{\prime} \Sigma^{-1} A\right)^{-} A^{\prime}, n-\rho(C)-p+\rho(A)\right)$.

In univariate linear models and the ordinary multivariate analysis of variance model the residuals are independent. Therefore it is of interest to see how the residuals $R_{1}, R_{2}$ and $R_{3}$ are related to the estimated mean structure $A \hat{B} C$.

THEOREM 3.3. Let $R_{1}, R_{2}$ and $R_{3}$, respectively be given by (2.1), (2.2) and (2.3), and let $A \hat{B} C$ be as in (1.4). Then $C\left[R_{i}, A \hat{B} C\right]=0, i=1,2$, and

$$
\begin{aligned}
C\left[R_{3}, A \hat{B} C\right]=-\frac{p-\rho(A)}{n-\rho(C)-p+\rho(A)-1} C^{\prime}\left(C C^{\prime}\right)^{-} C \otimes A\left(A^{\prime} \Sigma^{-1} A\right)^{-} A^{\prime} \\
\text { if } n-\rho(C)-p+\rho(A)-1>0 .
\end{aligned}
$$

Proof. Since $C\left[R_{i}, A \hat{B} C\right]=C\left[R_{i}, A B C\right], i=1,2$, the first part is proved. The second part follows from a result in Grizzle and Allen (1969) concerning the dispersion matrix for $\hat{B}$ since

$$
C\left[R_{3}, A \hat{B} C\right]=C\left[X C^{\prime}\left(C C^{\prime}\right)^{-} C, A \hat{B} C\right]-D[A \hat{B} C]
$$

and

$$
\begin{aligned}
C\left[X C^{\prime}\left(C C^{\prime}\right)^{-} C, A \hat{B} C\right] & =D\left[X C^{\prime}\left(C C^{\prime}\right)^{-} C\right]\left(I \otimes E\left[S^{-1} A\left(A^{\prime} S^{-1} A\right)^{-} A^{\prime}\right]\right. \\
& =\left(C^{\prime}\left(C C^{\prime}\right)^{-} C \otimes \Sigma\right)\left(I \otimes \Sigma^{-1} A\left(A^{\prime} \Sigma^{-1} A\right)^{-} A^{\prime}\right) \\
& =C^{\prime}\left(C C^{\prime}\right)^{-} C \otimes A\left(A^{\prime} \Sigma^{-1} A\right)^{-} A^{\prime} .
\end{aligned}
$$

Theorem 3.3 establishes that $R_{i}, i=1,2$ and $A \hat{B} C$ are uncorrelated but in the same manner as it was shown that the residuals are not independent it can also be shown that $\hat{R}_{i}, i=1,2$, and $A \hat{B} C$ are not independent.

In the next theorem we are going to obtain the dispersion matrices for the residuals. These give then some ideas about the randomness among the elements in $R_{i}$ and then, to some extent, can be used to identify extreme observations.

THEOREM 3.4. Let $R_{1}, R_{2}$ and $R_{3}$, respectively be given by (2.1), (2.2) and (2.3). Then if $n-\rho(C)-p+\rho(A)-1>0$ 
(i) $D\left[R_{1}\right]=\left(I-C^{\prime}\left(C C^{\prime}\right)^{-} C\right) \otimes \Sigma-c_{1}\left(I-C^{\prime}\left(C C^{\prime}\right)^{-} C\right) \otimes A\left(A^{\prime} \Sigma^{-1} A\right)^{-} A^{\prime}$,

(ii) $D\left[R_{2}\right]=c_{1}\left(I-C^{\prime}\left(C C^{\prime}\right)^{-} C\right) \otimes A\left(A^{\prime} \Sigma^{-1} A\right)^{-} A^{\prime}$, where

(iii) $D\left[R_{3}\right]=C^{\prime}\left(C C^{\prime}\right)^{-} C \otimes \Sigma-c_{2} C^{\prime}\left(C C^{\prime}\right)^{-} C \otimes A\left(A^{\prime} \Sigma^{-1} A\right)^{-} A^{\prime}$

$$
\begin{aligned}
c_{1} & =\frac{n-\rho(C)-p+\rho(A)}{n-\rho(C)}, \\
c_{2} & =\frac{n-\rho(C)-2(p-\rho(A))-1}{n-\rho(C)-p+\rho(A)-1} .
\end{aligned}
$$

Proof. Since $Y_{1}$ and $Y_{2} Y_{1}^{\prime}\left(Y_{1} Y_{1}^{\prime}\right)^{-1} Y_{1}$ are uncorrelated straightforward calculations yield

$$
D\left[R_{1}\right]=D\left[\Sigma^{1 / 2} \Gamma_{1}^{\prime} Y_{1}\right]+D\left[\Sigma^{1 / 2} \Gamma_{2}^{\prime} Y_{2} Y_{1}^{\prime}\left(Y_{1} Y_{1}^{\prime}\right)^{-1} Y_{1}\right] .
$$

Now,

$$
D\left[\Sigma^{1 / 2} \Gamma_{1}^{\prime} Y_{1}\right]=\left(I-C^{\prime}\left(C C^{\prime}\right)^{-} C\right) \otimes \Sigma^{1 / 2} \Gamma_{1}^{\prime} \Gamma_{1} \Sigma^{1 / 2}
$$

and

$$
D\left[\Sigma^{1 / 2} \Gamma_{2}^{\prime} Y_{2} Y_{1}^{\prime}\left(Y_{1} Y_{1}^{\prime}\right)^{-1} Y_{1}\right]=E\left[Y_{1}^{\prime}\left(Y_{1} Y_{1}^{\prime}\right)^{-1} Y_{1}\right] \otimes \Sigma^{1 / 2} \Gamma_{2}^{\prime} \Gamma_{2} \Sigma^{1 / 2} .
$$

If using (3.4) and that

$$
E\left[Y_{1}^{\prime}\left(Y_{1} Y_{1}^{\prime}\right)^{-1} Y_{1}\right]=\frac{p-\rho(A)}{n-\rho(C)}\left(I-C^{\prime}\left(C C^{\prime}\right)^{-} C\right),
$$

$D\left[R_{1}\right]$ in (i) is established.

$D\left[R_{2}\right]$ in (ii) is obtained since $R_{1}$ and $R_{2}$ are uncorrelated and $R_{1}+R_{2}=$ $X\left(I-C^{\prime}\left(C C^{\prime}\right)^{-} C\right)$. For $D\left[R_{3}\right]$ in (iii) we note that

$$
\begin{aligned}
D\left[R_{3}\right]=C^{\prime}\left(C C^{\prime}\right)^{-} C \otimes & \left\{\Sigma^{1 / 2} \Gamma_{1}^{\prime} \Gamma_{1} \Sigma^{1 / 2}\right. \\
& \left.+\Sigma^{1 / 2} \Gamma_{2}^{\prime} E\left[Y_{2} Y_{1}^{\prime}\left(Y_{1} Y_{1}^{\prime}\right)^{-1}\left(Y_{1} Y_{1}^{\prime}\right)^{-1} Y_{1} Y_{2}^{\prime}\right] \Gamma_{2} \Sigma^{1 / 2}\right\}
\end{aligned}
$$

and some calculations together with (3.4) establishes the theorem.

If the intention is to apply the results in Theorem 3.4 we have to estimate $D\left[R_{i}\right] i=1,2,3$. Unbiased estimators are given in the next theorem.

ThEOREM 3.5. Let $D\left[R_{i}\right], i=1,2,3$ be given in Theorem 3.4. The following estimators are unbiased.

(i) $\hat{D}\left[R_{1}\right]=\left(I-C^{\prime}\left(C C^{\prime}\right)^{-} C\right) \otimes \hat{\Sigma}$

$$
+\left(\frac{\rho(C)}{n} c_{2}-c_{1}\right) \frac{n}{n-\rho(C)-p+\rho(A)}\left(I-C^{\prime}\left(C C^{\prime}\right)^{-} C\right) \otimes A\left(A^{\prime} \hat{\Sigma}^{-1} A\right)^{-} A^{\prime},
$$

(ii) $\hat{D}\left[R_{2}\right]=\frac{n}{n-\rho(C)}\left(I-C^{\prime}\left(C C^{\prime}\right)^{-} C\right) \otimes A\left(A^{\prime} \hat{\Sigma}^{-1} A\right)^{-} A^{\prime}$,

(iii) $\hat{D}\left[R_{3}\right]=C^{\prime}\left(C C^{\prime}\right)-C \otimes\{\hat{\Sigma}$

$$
\left.+\left(\frac{\rho(C)}{n} c_{2}-c_{2}\right) \frac{n}{n-\rho(C)-p+\rho(A)} C^{\prime}\left(C C^{\prime}\right)^{-} C \otimes A\left(A^{\prime} \hat{\Sigma}^{-1} A\right)^{-} A^{\prime}\right\} .
$$


Proof. For this theorem it is utilized that

$$
E[\hat{\Sigma}]=\Sigma-\frac{\rho(C) c_{2}}{n} A\left(A^{\prime} \Sigma^{-1} A\right)^{-} A^{\prime}
$$

which was obtained by von Rosen (1990). Furthermore,

$$
\begin{aligned}
& n A\left(A^{\prime} \hat{\Sigma}^{-1} A\right)^{-} A^{\prime} \\
& \quad=A\left(A^{\prime} S^{-1} A\right)^{-} A^{\prime} \sim W_{p}\left(A\left(A^{\prime} \Sigma^{-1} A\right)^{-} A^{\prime}, n-\rho(C)-p+\rho(A)\right)
\end{aligned}
$$

and combining these two results verifies the theorem.

\section{Acknowledgements}

The support from the Swedish Natural Research Council is gratefully acknowledged.

\section{REFERENCES}

Belsley, D. A., Kuh, E. and Welsch, R. E. (1980). Regression Diagnostics: Identifying Influential Data and Sources of Collinearity, Wiley, New York.

Cook, R. D. and Weisberg, S. (1982). Residuals and Influence in Regression, Chapman \& Hall, New York.

Grizzle, J. E. and Allen, D. M. (1969). Analysis of growth and dose response curves, Biometrics, 25, 357-381.

Magnus, J. R. and Neudecker, H. (1979). The commutation matrix: Some properties and applications, Ann. Statist., 7, 381-394.

Potthoff, R. F. and Roy, S. N. (1964). A generalized multivariate analysis of variance model useful especially for growth curve problems, Biometrika, 51, 313-326.

Seber, G. A. F. (1984). Multivariate Obsrvations, Wiley, New York.

Srivastava, M. S. and Khatri, C. G. (1979). An Introduction to Multivariate Statistics, North Holland, New York.

Tobing, H. and McGilchrist, C. A. (1992). Recursive residuals for multivariate regression models, Austral. J. Statist., 34, 217-232.

von Rosen, D. (1989). Maximum likelihood estimators in multivariate linear normal models, $J$. Multivariate Anal., 31, 187-200.

von Rosen, D. (1990). Moments for a multivariate linear model with an application to the Growth Curve model, J. Multivariate Anal., 35, 243-259.

von Rosen, D. (1991). The Growth Curve model: A review, Comm. Statist. Theory Methods, 20, 2791-2822.

von Rosen, D. (1994). A study of residuals in the Growth Curve model, Research Report, No. 1994:36, Department of Mathematics, Uppsala University, Sweden.

Woolson, R. F. and Leeper, J. D. (1980). Growth curve analysis of complete and incomplete longitudinal data, Comm. Statist. Theory Methods, 9, 1491-1513. 